\title{
La promotion de l'empowerment et de l'entrepreneuriat féminin auprès des femmes exclues des politiques sociales et économiques au Sénégal
}

\author{
Ndèye Faty Sarr ${ }^{\mathrm{a}}$, Marie Fall ${ }^{\mathrm{b}}$
}

\begin{abstract}
RÉSUMÉ. Les catégories de femmes entrepreneures ne sont pas toutes homogènes. Dans le cadre des stratégies de promotion de l'entrepreneuriat féminin, les femmes peuvent faire face aux mêmes contraintes d'accès à des ressources, mais ne les vivent pas avec la même intensité. Au Sénégal, les inégalités de genre sont peu ou pas prises en considération dans les stratégies nationales de promotion sociale et économique des femmes. Ainsi, les besoins de certaines femmes qui s'activent dans de très petites entreprises ou qui mènent des activités économiques de petite envergure et générant de faibles revenus ne sont pas pris en compte lors de l'élaboration de ces stratégies nationales. Or, elles doivent bénéficier d'un encadrement vertical et horizontal renforcé qui tienne compte de leurs besoins spécifiques; et ce, dans le but de les intégrer au tissu économique et social national afin d'en faire des actrices du développement.
\end{abstract}

\begin{abstract}
Women entrepreneur categories are not all homogeneous. As part of strategies to promote female entrepreneurship, women may face the same constraints in accessing resources, but they do not experience them with the same intensity. In Senegal, gender inequalities are little or not taken into consideration in national strategies for the social and economic advancement of women. For example, the needs of some women in very small businesses or for small economic activities that generate low incomes are not considered in the development of these national strategies. Thus, they must benefit from a reinforced vertical and horizontal framework that takes into account their specific needs; however, they must benefit from a reinforced vertical and horizontal framework that takes into account their specific needs. This is required to integrate them into the national economic and social fabric in order to make them development players.
\end{abstract}

\section{Introduction}

En Afrique subsaharienne, les femmes sont de plus en plus impliquées dans des activités génératrices de revenus. Au Sénégal, l'intensification des activités entrepreneuriales est assez marquée. Toutefois, elle occulte la nature réelle des entreprises investies par les femmes. En effet, les Sénégalaises ont apporté une réponse circonstanciée pour faire face aux effets des programmes d'ajustement structurel (PAS) ${ }^{1}$ en s'investissant en masse dans des activités génératrices de revenus (AGR) (Simen et Diouf, 2014). Elles sont très nombreuses à s'investir dans de très petites AGR qui se résument le plus souvent à des activités commerciales avec de faibles revenus pour assurer la survie du ménage. Une étude menée par le Bureau international du travail (2020) sur le secteur informel révèle que $85,7 \%$ des femmes entrepreneures de ce secteur ont un chiffre d'affaires inférieur à 100000 francs CFA, soit 171 \$ US par mois. Quant aux femmes employées dans les entreprises informelles, elles gagneraient moins de 37000 francs CFA, soit $63 \$$ US par mois.

Quoique ces revenus mensuels soient faibles, plusieurs recherches ont montré qu'ils permettent une amélioration de la qualité de vie des ménages. En effet, l'acquisition de revenus financiers par les

\footnotetext{
${ }^{a}$ Maitresse de conférences en sociologie, chercheuse associée, Laboratoire d'études et de recherches appliquées sur l'Afrique, Université du Québec à Chicoutimi

${ }^{\mathrm{b}}$ Professeure agrégée, responsable, Laboratoire d'études et de recherches appliquées sur l'Afrique, Université du Québec à Chicoutimi
} 
femmes, même faibles, serait associée à une meilleure prise en charge de l'éducation, de l'alimentation et de la santé des enfants (Sarr, 2015). Ce lien de cause à effet aurait même justifié la promotion de la microfinance auprès des clientèles féminines. Ainsi, même si les AGR ont participé à l'amortissement des effets sociaux des PAS, les femmes peinent à se hisser en une force véritablement productive.

Partant de ce constat, nous cherchons à présenter les obstacles qui bloquent le passage des AGR d'une économie de survie à une économie à forte valeur ajoutée. Autrement dit, nous montrerons les obstacles qui entravent l'augmentation de leur niveau de productivité, leur efficacité et leur progression.

\section{Cadre théorique}

Nous mobiliserons le concept d'entrepreneure et celui d'empowerment pour mieux mettre en lumière l'importance de l'entrepreneuriat dans l'autonomisation des femmes qui s'activent dans de petites AGR.

\subsection{Le concept d'entrepreneure}

La femme entrepreneure africaine recherche l'épanouissement personnel, l'autonomie financière et la maitrise de son existence grâce au démarrage et à la gestion de sa propre entreprise (Belcourt et collab., 1991). Ali (2018) parle de «différences dans leurs motivations à démarrer leur entreprise, dans leurs compétences administratives et entrepreneuriales, et dans le contexte professionnel et les problèmes auxquels elles font face» (p. 27, trad. libre). L'Université coopérative internationale (UCI) a quant à elle dressé un portrait des entrepreneures en Afrique à la suite d'une mission d'intervention auprès de certaines femmes au Mali. Il s'agit de la personnalité, de la responsabilité, des relations personnelles, du sens des affaires, de la confiance, de la philosophie qui sous-tend l'esprit entrepreneurial et la compétence (Wane, 2009).

Depuis quelques années, la notion d'entrepreneure de nécessité est utilisée pour qualifier les activités économiques des femmes. En effet, selon la théorie de la formation de l'événement entrepreneurial de Shapero et Sokol (1982), la décision de créer une entreprise est le résultat d'un changement dans la situation personnelle de l'individu. Ce changement, qui est généré par un événement positif ou négatif, donne naissance à l'intention de créer une entreprise individuelle. Selon que ce changement est perçu positivement ou négativement, il se traduira par des motivations de création de nécessité dans le cas d'un changement perçu négativement ou d'opportunité dans le cas d'un changement perçu positivement (Giacomin et collab., 2016).

\subsection{Le concept d'empowerment}

Il existe une pluralité de définitions de l'empowerment. Globalement, les théoriciennes et théoriciens s'accordent sur son caractère multidimensionnel et sur le fait que l'empowerment dérive d'une situation de prise de conscience [des femmes] de leur situation de dominées, assortie d'une volonté individuelle ou collective de changer les inégalités de genre. Cette prise de conscience résulterait d'un retour sur soi ou d'une « activité autoréflexive» menée par les personnes dominées. Les théoriciennes et théoriciens s'accordent aussi sur l'idée que l'empowerment est liée à l'action. En effet, dans la plupart des définitions de ce concept, il apparait nettement que c'est par l'action qu'on envisage le processus d'empowerment (Le Bossé, 1996).

Pour sa part, Dagenais (1994) définit l'empowerment comme un processus bidimensionnel, c'est-à-dire à la fois individuel et collectif. Cependant, la dimension collective est peu considérée par les agences de développement international, qui présentent plutôt l'empowerment des femmes et des pauvres sous le seul angle de la dimension individuelle. Elles passent ainsi sous silence le rôle de surdétermination des structures macrosociales sur les situations de subordination (dans le cas des femmes) et de précarité (dans le cas des pauvres).

Quant à Mayoux (2001), elle conçoit l'empowerment comme un processus multidimensionnel qui traduit l'acquisition par les femmes de quatre piliers du pouvoir. D'abord, le «pouvoir en » réfère au changement d'optique sur le plan individuel. Le «pouvoir de» traduit la capacité de mobilisation collective des femmes et, au besoin, avec des hommes, qui renvoie au «pouvoir avec ». Finalement, «le pouvoir sur» concerne la perspective globale de remettre en question le système patriarcal. En outre, Mayoux (2001) considère qu'il existe trois types d'emponverment: économique individuelle, par l'amélioration du bien-être, et sociale et politique.

Molyneux (1985) considère que le processus d'empowerment comporte deux aspects : la satisfaction des besoins pratiques des femmes, qui correspondent 
à leurs besoins quotidiens de base, et celui de leurs intérêts stratégiques, qui sont liés à leur position sociale et aux relations de genre (Molyneux, 1985, cité dans Parpart, Connelly et Barriteau, 2000, cités dans Langevin, 2009).

Young (1998) abonde dans le même sens. Selon elle, il importe de préciser les deux composantes de l'empowerment, soit la condition des femmes et leur position sociale. En référence aux modèles d'analyse proposés par Moser et Molyneux (1985), la condition de la femme correspond aux besoins pratiques des femmes (c.-à-d. leurs conditions matérielles de vie), tandis que la position sociale de la femme réfère à son statut social relativement à l'homme ou aux hommes (Young, 1988, citée dans Langevin, 2009).

Selon Dagenais (1994), une analyse du processus d'empowerment des femmes doit tenir compte du fait que ces dernières ont des besoins pratiques à satisfaire, lesquels relèvent de l'empowerment économique : la nourriture, la santé, un cadre de vie de qualité, l'accès à un revenu décent. De son côté, l'empowerment sociale et politique réfère aux intérêts stratégiques de genre, c'est-à-dire l'analyse des bases matérielles de la répartition des rôles en fonction du genre. Cette distinction permet de montrer que toute politique visant l'empowerment globale (c.-à-d. économique, sociale et politique) ne peut faire fi de l'appropriation des intérêts stratégiques des femmes, qui passe par une remise en cause des structures de légitimation de leur statut inférieur.

Kabeer (1999) soutient que l'empowerment relève d'un changement social radical porté par des femmes qui, à un moment de leur existence, ont eu conscience de leur statut au sein de la société et ont cherché à le transformer. Cette auteure conçoit l'empowerment comme la capacité de la femme de défier les structures traditionnelles afin d'imposer sa propre vision des choses. Cette capacité se mesure par l'accès aux ressources et par le pouvoir de décision. Finalement, le tout se traduit par l'observation de résultats positifs en lien avec l'amélioration du bien-être. Fondamentalement, le concept d'empowerment réfère :

[...] au processus à partir duquel ceux à qui on a nié la capacité de prendre des décisions acquièrent cette habileté. En d'autres mots, l'emponverment implique un processus de changement. Les personnes qui doivent faire beaucoup de choix dans leur vie peuvent être très puissantes, mais elles ne sont pas autonomisées dans le sens avec lequel j'utilise ce mot, parce qu'au départ on ne leur a jamais enlevé cette habileté (Kabeer, 1999, p. 437 trad. libre).

L'empowerment serait aussi un «processus d'accès à plus de pouvoir. La notion de pouvoir étant entendue au sens large (économique, social et politique)» (Guérin et Palier, 2007p. 76).

Considérant les différentes définitions ci-haut présentées, les femmes qui s'activent dans de très petites entreprises commerciales ont besoin d'être accompagnées pour mieux s'intégrer dans le marché. Autrement dit, le contexte de précarité doit servir de levier à leur sens des initiatives. Toutefois, ces femmes, le plus souvent démunies, sont à la fois dépassées par les structures macrosociales et par les situations de subordination en raison de leur statut précaire. À ce titre, elles ont besoin d'être « autonomisées ». L'empowerment de ces femmes est une condition à la valorisation de leurs activités économiques.

\section{Le secteur informel : réceptacle des activités entrepreneuriales des Sénégalaises}

Les Sénégalaises sont réputées pour avoir la fibre entrepreneuriale. Déjà, à l'époque coloniale, les femmes de Saint-Louis du Sénégal étaient bien impliquées dans le commerce de la gomme arabique (Bonnardel, 1992). La dynamique de l'entrepreneuriat féminin s'est poursuivie après l'indépendance du pays en 1960. Cependant, la permanence de la crise de l'emploi consécutive à l'imposition des PAS à la suite des accords de Bretton Woods a amené les femmes à s'investir en masse dans les activités entrepreneuriales afin d'assurer la survie des ménages. Dans ce contexte, la nécessité explique l'engagement des femmes dans la réalisation de modestes AGR. Leurs activités professionnelles vont davantage se concentrer dans le secteur informel. Des femmes entrepreneures étaient bien décidées à sortir des sentiers battus. Elles ont été à l'origine des structures d'accumulation du capital et des pratiques économiques innovantes qui ont constitué une articulation entre une logique individuelle et une logique collective d'entrepreneuriat acceptable pour les communautés d'appartenance (Sarr, 1998). Dans plusieurs collectivités, les femmes sont au cœur des Fédérations locales de Groupements d'intérêt économique. Des leaders féminins sont présentées 
comme des modèles de réussite sociale et économique (Boulanger et Fall, 2020). Aujourd'hui, l'entrepreneuriat féminin est encouragé au Sénégal par la Délégation Générale à l'Entreprenariat Rapide des Femmes et des Jeunes.

Selon le Recensement général des entreprises (RGE) de l'Agence nationale de la statistique et de la démographie (ANSD), le tissu économique sénégalais est composé presque exclusivement d'entreprises informelles $(97 \%)$, avec une surreprésentation des activités de commerce (52\%) (ANSD, 2017). Le secteur informel regrouperait deux types d'activités économiques : les activités de subsistance, avec des entreprises de petite taille, et les entreprises de nécessité, caractérisées par la vulnérabilité, la précarité et l'absence de croissance (BIT, 2020). Les femmes formeraient $75 \%$ du secteur informel, selon le rapport annuel 2015-2016 d'ONU Femmes (2016). En effet, la faiblesse des barrières à l'entrée du secteur informel favoriserait l'entrée d'acteurs économiques disposant de peu de qualifications professionnelles et ayant un niveau de scolarité relativement bas. C'est le cas de la plupart des femmes.

Toutefois, le fait que ces dernières choisissent les activités commerciales dans le secteur informel ne s'explique pas seulement par la facilité avec laquelle on y entre. À la porosité du secteur informel s'ajoute sa grande capacité d'absorption d'un aréopage d'activités à très faible valeur ajoutée. Ainsi, le secteur informel devient le champ d'activité par excellence des femmes, premières victimes des crises sociales et économiques. Cela dit, dans l'observation de l'intensification du travail productif des femmes des pays d'Afrique subsaharienne, dans un contexte marqué par une précarité structurelle et infrastructurelle, il faut bien tenir compte des réalités spécifiques des femmes évoluant dans de très petites activités commerciales.

\section{Un état des lieux des dispositifs financiers dédiés aux femmes entrepreneures du secteur informel}

L'État du Sénégal s'est montré très engagé dans la promotion de l'entrepreneuriat féminin en créant en 2002 la Direction de l'entrepreneuriat des femmes. Sa feuille de route repose sur le développement d'actions permettant d'accompagner les femmes souhaitant créer une entreprise par divers services financiers et non financiers (formation en comptabilité, en commerce national et international; etc.) (Wane, 2009). L'actuel président de la république du Sénégal a également lancé la Délégation à l'entrepreneuriat rapide (DER), qui vise principalement les femmes et les jeunes. La Stratégie nationale pour le développement de l'entrepreneuriat féminin, qui souhaite renforcer le pouvoir économique des femmes ainsi que leur autonomisation afin de lutter contre la pauvreté, a aussi été élaborée. Elle met l'accent sur le rôle primordial des petites et moyennes entreprises (PME) dans la densification et la vitalité du tissu économique sénégalais, notamment dans la création d'emplois et de richesses (BIT, 2020). Dans les faits, les politiques et les programmes de promotion de l'entrepreneuriat féminin entrent dans une stratégie globale qui vise la formalisation des PME du secteur informel.

\section{Du financement existant, mais peu efficace pour l'empowerment des femmes}

Quelle que soit l'entreprise, le capital financier reste la ressource la plus importante pour réaliser des activités rentables. Toutefois, l'accès aux ressources financières reste très inégalitaire. En effet, beaucoup de recherches montrent les contraintes des femmes entrepreneures pour acquérir des ressources financières (Wane, 2009). L'intensité de cette problématique est variable selon les catégories de femmes entrepreneures. En effet, certaines d'entre elles, ciblées par des politiques et des programmes spécifiques, peuvent transcender ces contraintes. En revanche, les problèmes d'accès aux ressources financières demeurent pour les autres femmes.

Sous ce rapport, les systèmes traditionnels de mobilisation de l'épargne, notamment les tontines, constituent des recours indéniables. Développée dans les pays d'Afrique noire, la tontine consiste à collecter l'épargne à l'échelle des organisations de femmes. L'épargne collective permet ainsi de financer les besoins ponctuels d'une personne ou d'un groupe (Allemand, 2007). Comme le signalent Boyé et ses collaborateurs (2006), le principe des tontines est que les membres se réunissent à intervalles réguliers, généralement une fois par semaine. Chacune dépose dans la caisse commune un montant discuté au préalable. Chaque semaine, un membre, selon un ordre établi, dispose de la totalité de la somme amassée. L'ordre des tours peut être 
fixé d'un commun accord par un responsable ou de manière aléatoire par tirage au sort ou, souvent, par des enchères (Fall, M. et al., 2020). Ce système ne comporte pas de rémunération de l'épargne ni de paiement d'un taux d'intérêt, sauf dans le cas des enchères, où le plus offrant paie une prime pour disposer de l'argent en premier (Boyé et collab., 2006). Cette forme de mobilisation de ressources financières que sont les tontines ne répond que partiellement aux besoins de financement des femmes. Par contre, elle est de nature à remplir leurs besoins d'intégration sociale. De plus, puisque les mises ne sont pas rémunérées et qu'il n'y a pas d'intérêts appliqués, les tontines constituent un bon compromis pour les femmes.

L'augmentation du travail productif des femmes n'a pas manqué d'attirer l'attention des acteurs du développement, qui s'emploient de diverses manières à accompagner les femmes dans leurs activités entrepreneuriales en leur offrant des lignes de crédit. C'est le cas des programmes de microfinance, qui ont été présentés dans les pays africains à la fois comme une politique de développement et comme un outil de lutte contre la pauvreté. Ce double objectif serait fondé sur un postulat central et sur un certain nombre de suppositions. En effet, les promoteurs de la microfinance estiment que l'instrumentalisation de la fonction économique des femmes, c'est-à-dire l'utilisation de leur dynamisme professionnel, serait un moyen efficace de lutter contre la pauvreté et un vecteur de changement social. Ces suppositions expliqueraient l'application d'une politique de développement à la fin des années 1990 dans les pays du Sud.

Malheureusement, après plusieurs années d'application dans différents pays du monde, plusieurs observations permettent de relever que la microfinance n'a pas tenu ses promesses de développement. En effet, aucune avancée majeure des activités commerciales des femmes n'a pu être observée de façon significative, alors même que ces catégories de femmes ont été les cibles spécifiques. Les taux d'intérêt jugés élevés appliqués aux micro prêts expliqueraient en partie leur inefficacité. En effet, des taux aussi prohibitifs appliqués à des activités productives de petite envergure n'ont pas vocation de permettre un décollage qualitatif des activités économiques. En réalité, le microcrédit s'insérerait dans un ensemble de stratégies de financement mobilisées par les femmes pour maintenir leurs activités.

L'observation du recours à deux modes d'accès aux ressources financières que sont les tontines et le microcrédit révèle l'existence de liens établis par les femmes entre le système traditionnel (informel) de mobilisation de l'épargne et le système moderne (formel) d'épargne et de crédit. Dans les faits, la présence des structures de microfinance suscite chez les femmes un effort de réadaptation. Les nouvelles possibilités de mobilisation des ressources financières ne viennent pas concurrencer les pratiques préexistantes. En effet, les nouvelles pratiques s'arriment aux pratiques préexistantes pour donner naissance à des pratiques hybrides mêlant tradition et modernité.

Ainsi, pour accéder à un premier prêt, les femmes peuvent recourir à plusieurs sources de financement pour mobiliser la caution exigée par le bailleur, notamment les tontines et d'autres prêts de membres du réseau social. Toutefois, les activités commerciales financées à partir de cette pluralité de sources peinent à décoller.

\section{Des formations en entrepreneuriat déficientes}

La gestion d'une entreprise implique la maitrise d'un certain nombre de compétences (gestion, marketing, comptabilité.) Or, les femmes entrepreneures présentent un taux d'analphabétisme assez élevé résultant d'une faible scolarisation. Certes, des campagnes d'alphabétisation ont été instaurées par les services de l'État sénégalais et les partenaires techniques et financiers ; mais elles ne sont pas intégrées à des programmes de formation pour une meilleure création et une tenue des entreprises.

D’un autre côté, si les programmes de microcrédit ont justifié le ciblage des femmes défavorisées pour la lutte contre la pauvreté, l'analyse de leurs pratiques révèle l'absence d'offres de services non financiers accompagnant l'octroi de crédits à ces femmes. L'objectif de la pérennisation et de la rentabilisation prend le dessus sur l'offre de services de base non financiers. 


\section{Les contraintes liées à l'entrepreneuriat féminin}

Les stratégies de promotion de l'entrepreneuriat féminin ont identifié un certain nombre de contraintes auxquelles les femmes entrepreneures (des secteurs formel et informel) font face. En effet, ces dernières éprouvent des difficultés liées à une connaissance optimale des réalités des marchés économiques et financiers. Les contraintes sont liées à l'éducation, à la formation en emploi, à l'accessibilité des structures d'appui et de conseil, à l'accès au financement ainsi qu'à l'accès effectif aux facteurs de production (Wane, 2009).

Au Sénégal, l'institution du mariage reste importante (Dial, 2008). Le partage genré des tâches au sein du ménage compromet l'investissement optimal des femmes dans les activités productives. En d'autres mots, les femmes sont dans l'obligation morale et sociale d'assumer les fonctions de reproduction. La problématique de la conciliation entre le travail et la gestion des tâches domestiques se pose ainsi aux femmes en des termes plus complexes. En raison de leur faible autonomie financière, les marges de manœuvre des femmes restent faibles. En effet, plusieurs études démontrent que cette division inégale du travail domestique constitue un véritable goulot d'étranglement qui amène les femmes entrepreneures à consacrer peu de temps à leur entreprise (Sarr, 2015). Qui plus est, la nature des activités entrepreneuriales des femmes africaines démontre que les domaines investis par ces dernières génèrent peu de valeur ajoutée, du fait qu'elles choisissent des activités entrepreneuriales qui constituent en réalité des prolongements de leur rôle traditionnel (Sarr, 2015).

Les contraintes mentionnées plombent les initiatives entrepreneuriales des femmes sénégalaises. Elles sont décuplées pour les femmes qui mènent de très petites activités commerciales sans un réel accès à des sources de financement sécurisé ni à une formation d'appoint pour obtenir des outils de gestion de leur entreprise.

\section{Conclusion}

Malgré les nombreuses contraintes auxquelles elles font face, les femmes entrepreneures sont des modèles de réussite sociale et économique au Sénégal L'État sénégalais et les partenaires au développement ont beaucoup investi dans la promotion de l'entrepreneuriat des femmes à travers plusieurs dispositifs de soutien financier et d'accompagnement personnalisé. Toutefois, ces dispositifs, qui reposent sur une stratégie globale de formalisation de l'économie, ciblent plutôt les femmes entrepreneures des PME du secteur informel. Or, ce secteur est majoritairement investi par des femmes qui s'activent dans des petites activités commerciales génératrices de revenus. Compte tenu des contraintes sociales et économiques, ces femmes doivent faire l'objet de mesures d'accompagnement adaptées à leurs conditions et à leurs besoins afin d'en faire des actrices du développement social et économique.

\section{NOTES}

1 «Les ajustements sont une réponse à la crise financière, notamment d'endettement, connue par les pays africains. Ils conduisent à des prêts sous conditionnalités qui ont pour objet premier de boucler les finances publiques. Ils se traduisent par un ensemble de réformes visant à retrouver les grands équilibres macro-économiques et financiers et à remettre l'économie sur un sentier durable de croissance. Leurs effets macro-économiques doivent être différenciés de leurs effets sociaux et redistributifs » (Hugon et Pagès, 1998).

2 Les outils linguistiques en français recommandent plutôt les termes «autonomisation », « habilitation », « responsabilisation », «capacitation» ou « renforcement». Nous adoptons ici le terme « empowerment».

\section{RÉFÉRENCES}

Agence nationale de la statistique et de la démographie (ANSD) (2017). Rapport global du Recensement général des entreprises 2016. http://www.ansd.sn/ressources/publications/Rapport\%20global-juil-2017.pdf

Ali, R. S. (2018). Feminist theory and its influence on female entrepreneur's growth intentions. International Journal of Innovation and Economic Development, 4(3), 20-31. https://doi.org/10.18775/ijied.1849-7551-7020.2015.43.2003

Allemand, S. (2007). La microfinance n'est plus une utopie! Éditions Autrement. 
Belcourt, M. L., Burke, R. J. et Lee-Gosselin, H. (1991). Une cage de verre : les entrepreneures an Canada. Conseil consultatif canadien sur la situation de la femme.

Boyé, S., Hajdenberg, J. et Poursat, C. (2006). Le guide la microfinance. Microcrédit et épargne pour le développement. Éditions d’Organisation.

Bonnardel, R. (1992). Saint-Louis du Sénégal : mort ou naissance. L'Harmattan.

Boulanger, N. et Fall, M. (dir) (2020). Fatou Sarr, l'égérie des îles du Saloum au destin exceptionnel. Entretiens avec Noémie Boulanger. GRIR (Groupe de recherche et d'intervention régionales), UQAC.

Bureau international du travail (BIT). (2020). Diagnostic de l'économie informelle du Sénégal. https://www.ilo.org/wcmsp5/groups/public/ ---ed_protect/---protrav/---travail/documents/publication/wcms_735752.pdf

Dagenais, H. (1994). Women, feminism anddevelopment. Canadian Research Institute for the Advancement of Women.

Dial, F. B. (2008). Mariage et divorce à Dakar : itinéraires féminins. Éditions Karthala.

Fall, M. Gouin-Bonenfant, M. Mbaye, E et Séne. B. (2020). Actes de la deuxième conférence internationale sur la Francophonie économique «L'entrepreneuriat et l'insertion professionnelle des jeunes et des femmes en Afrique francophone ». Université Mohammed V de Rabat, 2-4 mars 2020. https://ofe.umontreal.ca/fileadmin/ofe/ documents/Actes/Conf_OFE_CIRPEC_2019/Texte36.pdf

Giacomin, O., Janssen, F. et Guyot, J.-L. (2016). Entrepreneurs de nécessité et d’opportunité : quels comportements durant la phase de création? Revue de l'entrepreneuriat, 34(15), 181-204.https:/ /www.cairn.info/revue-de-l-entrepreneuriat-2016-3-page-181.htm

Guérin, I. et Palier, J. (2006). Microfinance et empowerment des femmes : la révolution silencieuse aura-t-elle lieu? Finance et Bien Commun, 2(2), 76-82. https://doi.org/10.3917/fbc.025.0076

Hugon, P. et Pagès, N. (1998). Ajustement structurel, emploi et rôle des partenaires sociaux en Afrique francophone. Cabiers de l'emploi et de la formation, 28. https://www.ilo.org/wcmsp5/groups/public/---ed_emp/documents/publication/wcms_120327.pdf

Kabeer, N. (1999). Resources, agency, achievements: Reflections on the measurement of women's empowerment. Development and Change, 30(3), 435-464.https://doi.org/10.1111/1467-7660.00125

Langevin, M. (2009). Les relations entre la participation aux programmes de microfinance et les processus d'empowverment des femmes [Mémoire de maitrise non publié, Université Laval]. Corpus UL. http://hdl.handle.net/20.500.11794/21190

Le Bossé, Y. (1996). Empowerment et pratiques sociales : illustration du potentiel d'une utopie prise au sérieux. Nowvelles pratiques sociales, 9(1), 127-145. https://doi.org/10.7202/301353ar

Mayoux, L. (2001). Vers un nouveau paradigme dans les programmes de microcrédit. Dans J. Bisilliat (dir.) Genre et économie : un premier éclairage (p. 325-331). L’Harmattan.

ONU Femmes. (2016). Rapport annuel 2015-2016.ONU Femmes. https://annualreport.unwomen.org/fr/2016

Sarr, F. (1998). Étude des pratiques de solidarité des entrepreneures issues du secteur informel : quelles perspectives pour les pratiques sociales? [Thèse de doctorat non publiée, Université Laval]. https://www.giersa.ulaval.ca/sites/giersa.ulaval.ca/files/memoires/document_133.pdf

Sarr, N.-D. (2015). Impacts des microcrédits de l’Union des Mutuelles pour la Mobilisation de l'Épargne et le Crédit (UMPAMECAS) sur les conditions économique et sociale des femmes : «empowerment» ou instrumentalisation ? [Thèse de doctorat, Université Laval]. CorpusUL. https://corpus.ulaval.ca/jspui/handle/20.500.11794/25759

Shapero, A. and Sokol, L. (1982). The Social Dimensions of Entrepreneurship. Dans Kent, C.A., Sexton, D.L. and Vesper, K.H., Encyclopedia of Entrepreneurship, (p. 72-90). Prentice-Hall, Engelwoods.

Simen, S. et Diouf, I. (2014). Entreprenariat féminin au Sénégal : vers un modèle entrepreneurial de «nécessité» dans les pays en développement. Revue ouest-africaine de sciences économiques et de gestion, 7(2). https://www.researchgate.net/ publication/325575470_Entreprenariat_feminin_au_Senegal_vers_un_modele_entrepreneurial_de_ necessite_dans_les_pays_en_developpement

Wane, A. (2009). L'entrepreneuriat féminin au Sénégal : obstacles et essais de solution [Mémoire de maitrise non publié, Université Cheikh Anta Diop] 